\title{
Preoperative evaluation, surgical strategy and long-term outcome of anorectal malformations in newborn period: Single center experience
}

\author{
Murat Sanal $^{\text {** }}$, Oliver Renz ${ }^{1}$, Paul Hechenleitner ${ }^{1}$ and Beatrice Häussler ${ }^{1}$ \\ ${ }^{1}$ Department of Pediatric Surgery, Clinic VTT, Innsbruck Medical University, Austria
}

\section{Introduction}

Anorectal malformations are congenital defects, where the lower end of digestive tract, including anus and rectum, do not develop properly.

The most widely accepted is Pena's classification. Anal stenosis or web, rectal atresia, rectoperineal fistula and imperforate anus without fistula are common for both male and female. Specific male defects are rectourethral bulbar, prostatic and bladderneck fistula. Specific female defects are rectovestibular fistula and cloaca with short or long common channel. The first description of anorectal malformations goes back to ancient times. Doing a stoma for discharge and/or protective purposes has been seen much later.

Introducing of posterior sagittal anorectoplasty as the choice of surgical treatment by Alberto Pena was one of the most important milestones in the treatment of anorectal malformations.

With this approach, the anatomical reconstruction of anal canal became much better.

Formation of divided colostomy represent the first stage in the surgical treatment of anorectal malformations.

However, the newborn management of rectoperineal and rectovestibular fistula consider primary repair with PSARP followed with TPN (total parenteral nutrition) for next 10 days as the choice of treatment. After stoma formation, high pressure distal colostogram should be performed in order to distinguish imperforate anus without fistula of rectourethral fistula and in the case of last one to determinate the type of rectourethral bulbar, prostatic or bladderneck fistula. Now, after establishing the proper diagnose we are ready for PSARP which can be performed within 2 to 3 months.

This study presents data of 42 babies with ARM including the preoperative evaluations, surgical procedures and the long-term outcome of these patients.

\section{Materials/methods}

The study of 42 ARM newborns, treated in our department between 2004 - 2016 was designed retrospectively.

Atotal number of 19 babies with perineal fistula underwent primary repair-anoplasty without a colostomy. We perform the anoplasty by lithotomy position, preparing the fistula circumferentially until to achieve normal rectum, marking of the external sphincter using muscle stimulator and pulled the rectum through the sphincter to its normal location.
Two males with rectobladderneck fistula, 5 patients with rectouretral prostatic fistula and 8 females with rectovestibular fistula underwent transverse diverting colostomy first and 4 to 6 weeks later PSARP.

There were 8 babies with imperforate anus without fistula. Among these 8 babies, 3 of them had a distance less than $1 \mathrm{~cm}$ between rectal gas and perineum, which was confirmed with $\mathrm{x}$-ray and sonography examinations. They underwent anoplasty without colostomy formation. Five patients of this group was treated with protective colostomy first.

The choice of protective colostomy we used in our patients was transverse diverting colostomy. Distal part of the colostomy was irrigated biweekly with normal saline solution.

All babies with colostomy underwent 4 to 6 weeks later PSARP to achieve the intestinal continuity.

14 days after simple anoplasty or PSARP, anal dilatation protocol started and continued about 7 months.For all children an urodynamic investigation was performed.

First year after definitive surgery follow up was made once a month. In the next five years follow up was biannual.

\section{Results}

The characteristics of the patients and distribution of sex are presented in Table 1.

In one female with imperforate anus, after simple anoplasty, severe wound infection and recurrence fistula occurred. This baby is treated with a protective colostomy and redo of anoplasty.

Four patients developed perianal wound infection in the early postoperative period. They were treated conservatively using wound dressing material mepitel (Safetac $c^{\varpi}$ Technologie). There was no need for colostomy revision.

One patient developed stoma prolapsus, which was easily reponible where,no revision was needed. Two patients have wound healings

Correspondence to: Murat Sanal, MD, Department of Pediatric Surgery, Clinic VTT, Innsbruck Medical University, Austria, Tel: + 43512504 80774, Fax: + 43512 504 25693,E-mail: murat.sanal@tirol-kliniken.at; alimuratsanal@gmail.com

Key words: anorectal malformations, posterosagittal anorectoplasty, anal atresia, anoplasty

Received: June 02, 2017; Accepted: June 20, 2017; Published: June 22, 2017 
Table 1. Patients characteristics $(\mathrm{N}=42)$.

\begin{tabular}{|c|c|c|}
\hline & Male & Female \\
\hline Perineal fistula & 12 & 7 \\
\hline İmperforated anus without fistula & 3 & 5 \\
\hline Rectobladderneck & 2 & - \\
\hline Rectouretral prostatic fistula & 5 & - \\
\hline Rectovestibular fistula & - & 8 \\
\hline
\end{tabular}

problems around the stoma and they were treated successfully with the help of our wound care team (Figure 1). Surgical complications are listed in Table 2.

One female with caudal regression syndrome need clean intermittent catheterization and from time to time because of severe chronic recurrent anal sores need hospital care (Figure 2). Her fecal incontinence was managed via daily wash-outs.

In the long term period have the patients satisfied bowel function with formed stools. The main problem with stool was constipation with fecal impaction, which needed wash-outs and rarely digital evacuation.

\section{Discussion}

Anorectal malformations consist of wide spectrum of defects that range from the simple anus imperforatus, treated easily via anoplasty with excellent functional results, to very complex anomalies, difficult to manage, are commonly associated with other anomalies and have a poor prognosis.

The average worldwide incidence is 1 in 5000 live births and slightly more common in boys. Boys have as likely as two times higher anomalies [1]. Imperforate anus may occur as unique malformation, however it coexists with several other anomalies such as duodenal atresia, tracheoesophageal fistula, renal and vertebral defects [2]. There are no exact cause of ARM. It may be due to failure of development of the cloacal membrane or failure of recanalization of the anal canal during embryonic life [3].

The history of ARM revealed us that the imperforate anus is well known since ancient time. The first medical documents from Şerafettin Sabuncuoğlu (1385-1468) showed the operative illustrations about this condition [4]. For a long time patients with high type atresia could not treated and survived. The first colostomy was performed in 1783 [5].

In course of time we have been seeing the development of sigmoid colostomy and then single-stage abdominoperineal procedures became popular [6,7]. After introduction of the procedure postero sagittal anorectoplasty (PSARP) changed the surgical treatment dramatically. Surgeons have to view the anatomy of these defects more clearly with the usage of PSARP and could repair them under direct vision [8,9].

The most important examination by ARM patients is the inspection of the perineal region, which gives us clues to the type of malformation. Hence we can make a desicion about a colostomy or a primary operation. Presence of the meconium on the perineum is evidence of perineal fistula and presence of the meconium in the urine confirms a rectourinary fistula.

Radiological examinations are also valuable to show us the choice of the treatment. A crucial point of the radiological evaluations is timing. An x ray before 24 hours is too early and may not show the real anatomy because the rectum is collapsed. During preoperative evaluation in case of no meconium is present on the perineum after 24 hours, a cross-table lateral $\mathrm{x}$-ray in prone position is very helpful in order to evaluate of the rectal gas [9]. Generally it is recommended to perform a protective colostomy, if the rectal gas does not detected beyond the coccyx.

In order to determine the distance between the rectal gas and perineum, our radiologists do an x-ray examination in collaboration with sonography. After this combined US \& x-ray examination, if the distance is less than $1 \mathrm{~cm}$, we choose to perform a simple anoplasty without a protective colostomy. If it is more than $1 \mathrm{~cm}$ we do a protective transversostomy.

Handling of the stoma bags and management of the stoma by newborns are not that easy because of the insufficient place. The most proper place for the stoma is upper abdomen according to our experience and that is why we prefer a diverting transversostomy as a protective stoma.

Evaluation of the ARM was done traditionally according to the nomenclature "high", "intermediate" and "low"defects. This is a worldwide used terminology, however an anatomic classification has more clinical value [9]. We practiced our surgical strategy algorithm to determine this important anatomical condition.

Table 2. Surgical complications.

\begin{tabular}{|c|c|c|c|}
\hline \multicolumn{2}{|c|}{ Stoma } & \multicolumn{2}{c|}{ Anus/Perineum } \\
\hline Retraction & - & Fistula recurrence & 1 \\
\hline Prolapse & 1 & Anal stricture & - \\
\hline Obstruction & - & Wound infection & 5 \\
\hline Parastomal hernia & - & & \\
\hline Need for revision & - & & \\
\hline Skin excoriations & 2 & & \\
\hline
\end{tabular}

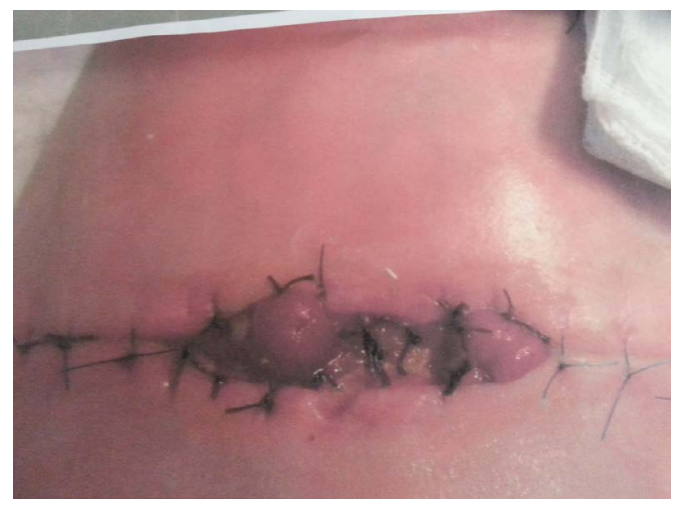

Figure 1. Severe parastomal wound infection.

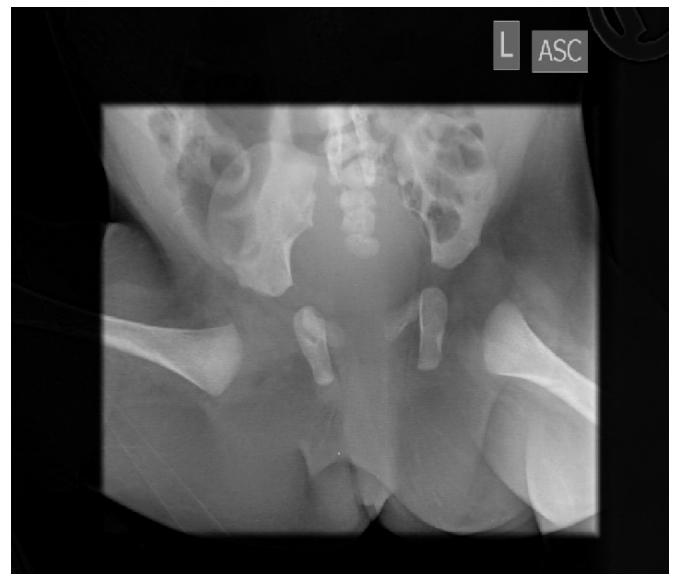

Figure 2. Abscence of the coccyx, caudal regression syndrome. 
Evaluation of the associated anomalies

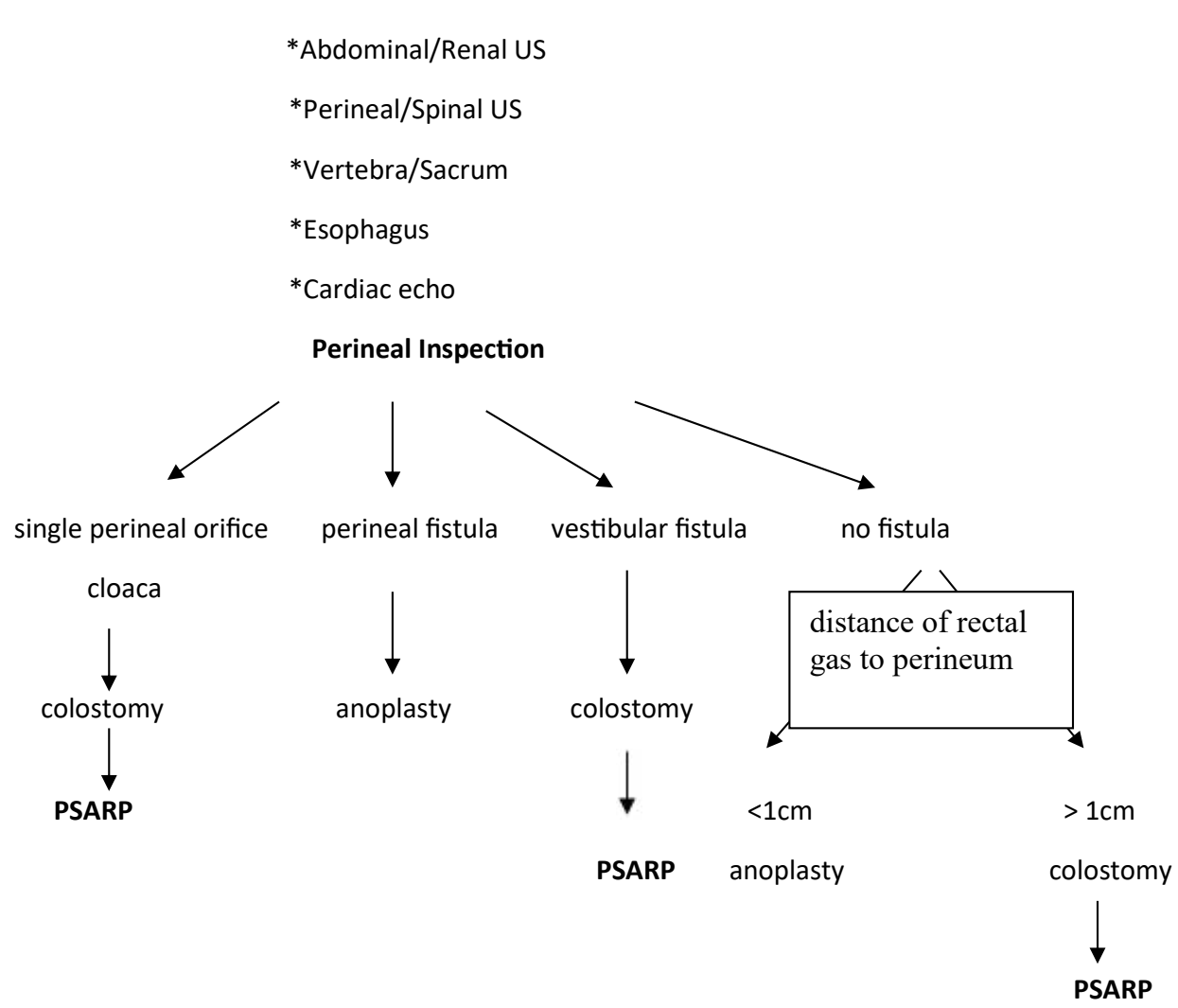

Table 3. Preoperative evaluation and surgical strategy.

After careful inspection of the perineum, in general we can make a decision whether we perform a colostomy or we can use a simple anoplasty. To determine the distance between rectal gas and perineum the examinations of our radiologists are very helpful (Table 3).

In the majority of ARM patients the sensation of the anal canal is not normal. The problem of the bowel motility and defecation is in low types less than the higher form. Soiling problems are usually related to constipation and when constipation is managed correctly, the soiling episodes disappear. On the other hand patients, who underwent PSARP have a tendency to develop megarectum. To minimize these complications, a careful operation and strict follow up are very valuable. We do not see megarectum in our series.

To our experience, anal dilatation protocol and wash outs throughout postoperative controls is very helpful to avoid the development of megarectum and anal stricture.

As a conclusion, ARM is a complex situation and have a high complications potential. To avoid these complications a careful preoperative physical examination, competent radiological assistance, meticulous proper operation and frequently careful postoperative controls are the clues to achieve a better result.

\section{Conflicts of interest}

This study has no potential conflict of interest.

\section{Acknowledgement}

We thank so much to our stoma care team, especially to Sr. Gerda

\section{References}

1. Falcone RA, Levitt MA, Peña A, Bates MD (2007) Increased heritability of certain types of anorectal malformations. J Pediatr Surg 42: 124-128. [Crossef]

2. Pinsky L (1978) The syndromology of anorectal malformation (atresia, stenosis, ectopia). Am J Med Genet 1: 461-474. [Crossref]

3. de Vries PA, Friedland GW (1974) The staged sequential development of the anus and rectum in humanembryos and fetuses. J Pediatr Surg 9:755-759. [Crossref]

4. Büyükünal SN, Sari N (1991) Serafeddin Sabuncuoğlu, the author of the earliest pediatric surgical atlas: Cerrahiye-i Ilhaniye. J Pediatr Surg 26: 1148-1151. [Crossref]

5. Littre A (1710) Diverses observations anatomiques, histoire de l'acadamemie royale de Science, Paris

6. Rhoads JE, Pipes RL, Randall JP (1948) A simultaneous abdominal and perineal approach in operations for imperforate anus with atresia of the rectum and rectosigmoid. Ann Surg 127: 552-556. [Crossref]

7. Norris WJ, Brophy TW, Brayton D (1949) Imperforate anus; a case series and preliminary report on the one stage abdominoperineal operation. Surg Gynecol Obstet 88: 623-634. [Crossref]

8. Rosen NG, Hong AR, Soffer SZ, Rodriguez G, Peña A (2002) Rectovaginal fistula: a common diagnostic error with significant consequences in girls with anorectal malformations. J Pediatr Surg 37: 961-965. [Crossref]

9. Derbew M, Levitt MA (2009) Newborn management of anorectal malformations. Surgery in Africa Review 4: 1-14.

Copyright: (C2017 Sanal M. This is an open-access article distributed under the terms of the Creative Commons Attribution License, which permits unrestricted use, distribution, and reproduction in any medium, provided the original author and source are credited. 